\title{
Application of a carbazole derivative as a spectroscopic fluorescent probe for real time monitoring of cationic photopolymerization
}

\author{
Joanna Ortyl", Mateusz Galica, Roman Popielarz, Dariusz Bogdał \\ Cracow University of Technology, Faculty of Chemical Engineering and Technology, Chair of Biotechnology and Physical \\ Chemistry, Warszawska 24, 31-155 Kraków, Poland \\ "Corresponding author: e-mail: jortyl@chemia.pk.edu.pl
}

\begin{abstract}
The performance of 1-(9-ethylcarbazol-3-yl)-4,4,4-trifluorobutane-1,3-dione (1) as a fluorescent probe for the monitoring of cationic photopolymerization processes by Fluorescence Probe Technique (FPT) has been evaluated in comparison with the response of 7-diethylamino-4-methylcoumarin (Coumarin 1) (2). Triethylene glycol divinyl ether and diphenyliodonium hexafluorophosphate were used as an example monomer and a cationic photoinitiator respectively. It has been found that the probe $\mathbf{1}$ withstands the cationic polymerization conditions and provides correct probe response. 1-(9-ethylcarbazol-3-yl)-4,4,4-trifluorobutane-1,3-dione shifts its fluorescence spectrum with progress of cationic photopolymerization of the monomer, which enables the monitoring of the polymerization progress using the fluorescence intensity ratio measured at two different wavelengths as the progress indicator. By comparing the behavior of $\mathbf{1}$ and $\mathbf{2}$, it has been documented that the fluorescence spectrum of probe $\mathbf{1}$ shows a spectacular hypsochromic shift $(\Delta \lambda=33 \mathrm{~nm})$ upon the monomer polymerization, while the shift of 2 is three times smaller $(\Delta \lambda=11 \mathrm{~nm})$. Moreover, the sensitivity of probe $\mathbf{1}$ is more than 2.5-times higher than that of any other probes suitable for monitoring cationic polymerization processes, reported previously. Therefore, application of the carbazole derivative (1) as a new probe for the monitoring of the crosslinking process of coatings cured by cationic photopolymerization has been proposed.
\end{abstract}

Keywords: spectroscopic fluorescent probe, FPT, fluorescence, carbazole, photopolymerization.

\section{INTRODUCTION}

Spectroscopic molecular probes have found a variety of uses as fluorescent probes ${ }^{1}$ and labels in many applications. For example, fluorescent probes are the most powerful tools that can be used for the measurement of solvent polarity and viscosity ${ }^{3}$, in biochemical applications ${ }^{4}$ and in material science ${ }^{5-7}$. Fluorescent molecular probes are usually used to understand physical and chemical processes that occur at a molecular level. This is possible because their fluorescence characteristics is sensitive to changes of polarity and/or mobility of the molecular environment where the probe molecules are located ${ }^{8-11}$. Moreover, the fluorescence spectroscopy has received considerable interest as an analytical tool due to its high sensitivity, selectivity and non-destructive characteristics ${ }^{11}$. In addition, remote sensing is readily implemented in fluorescence methods by the use of fiber-optic cables to transmit optical signals to and from the analytical site in real time ${ }^{5,6,12}$. In particular, polymerization kinetics of photocurable formulations can be precisely monitored in real-time using fluorescent molecular probes. This technique, commonly known as Fluorescence Probe Technology (FPT), has proven to be an extremely useful analytical tool in polymer chemistry ${ }^{13}$. One of the most important applications of fluorescence probes is for the monitoring progress of fast photopolymerization proces$\operatorname{ses}^{14,15}$, where other more conventional methods often fail. FPT provides valuable information on the kinetics of polymerization ${ }^{16}$, curing ${ }^{17}$ and crosslinking ${ }^{18}$ processes.

Theoretically every process that causes the change of the system polarity or microviscosity should be able to be monitored by FPT. However, depending on the type of the process, and the monitoring parameters, appropriate structure and characteristics of the probe are required ${ }^{\mathbf{1 1}}$. Therefore, there are no completely versatile probes. In fact, various fluorescent probes have been developed for monitoring the progress of polymerization or the final degree of cure of photocurable coatings ${ }^{11}$. Typical fluorescent probes used for free-radically cured coating formulations contain basic amino functionality in their structure, which is necessary to afford high fluorescence efficiency and a large shift of the fluorescence spectrum with the change of microviscosity and micropolarity of the probe environment. These probes are usually not useful for typical cationic cures, because they interfere with the polymerization reaction. Thus, there is a need for fluorescence probes which would be useful for cure monitoring of polymeric coatings or films cured by cationic polymerization.

In this communication the performance of a new fluorescent probe, based on carbazole skeleton, as a probe for monitoring cationic photopolymerization of monomers by Fluorescence Probe Technology (FPT) is reported in comparison with the performance of 7-diethylamino-4-methylcoumarin probe.

\section{EXPERIMENTAL}

\section{Materials}

Triethylene glycol divinyl ether (TEGDVE, Sigma Aldrich) and diphenyliodonium hexafluorophosphate (Alfa Aesar) were selected as a model monomer and a cationic polymerization photoinitiator, respectively. 1-(9-ethylcarbazol-3-yl)-4,4,4-trifluorobutane-1,3-dione (1) was selected for the role of a fluorescent probes in this study, while 7-diethylamino-4-methylcoumarin (2) (Sigma-Aldrich), which is a typical probe used for free radical polymerization, was selected as a reference probe for comparison.

All reagents used for synthesis of the probe $\mathbf{1}$ were of analytical grade. The solvents were dried by standard methods. 9-ethylcarbazole, ethyl trifluoroacetate and 
acetyl chloride were purchased from Sigma Aldrich. The quality of reaction products was measured with the help of HPLC-ESI-MS analysis (Agilent 1200 Series LC 6210 TOF MS with ESI source). NMR spectra were recorded on a Varian Mercury-VX $300 \mathrm{MHz}$ spectrometer. Fourier transform infrared spectra (FTIR) were recorded in $\mathrm{KBr}$ pellets using Biorad FTS FTIR spectrometer. The progress of synthesis was monitored by thin layer chromatography (TLC) (Silicagel GF-254 from Merck; eluent: hexane/acetone/bromoform $=10: 1: 2$ ), using a UV lamp for product detection. The melting point was determined using the BarnSteadt Electrothermal 9200 apparatus.

\section{Synthesis of 1-(9-ethylcarbazol-3-yl)-4,4,4-trifluorobuta-} ne-1,3-dione (1)

A general scheme of the synthesis of $\mathbf{1}$ is presented in Figure 1. It is based on the two-step procedure originally proposed by Bowyer and Pei $\mathrm{He}^{19,20}$.

Our procedure was similar to that described in literature, but we used different reaction conditions and a different catalyst. The acylation of 9-ethylcarbazole $(4.0 \mathrm{~g}, 20.5 \mathrm{mmol})$ was conducted by the dropwise addition of acetyl chloride $(1.80 \mathrm{~mL}, 24.5 \mathrm{mmol})$ into 9-ethylcarbazole solution in dichloromethane in the presence of anhydrous $\mathrm{AlCl}_{3}(3.4 \mathrm{~g}, 25.0 \mathrm{mmol})$ at the temperature $0-10^{\circ} \mathrm{C}$ under argon atmosphere. Next, the reaction mixture was stirred at ambient temperature for about $24 \mathrm{~h}$, and then cold water $(100 \mathrm{~mL})$ was added. The lower organic layer was separated, and the aqueous phase was extracted with $\mathrm{CH}_{2} \mathrm{Cl}_{2}(3 \times 20 \mathrm{~mL})$. The organic layer was combined with the extracts and was dried over anhydrous $\mathrm{MgSO}_{4}$. Methylene chloride was removed by rotary evaporation and the residue was purified by column chromatography on silica gel using a mixture of ethyl acetate/petroleum ether (1:9) as an eluent to yield 1-(9-ethylcarbazol-3-yl)ethanone (1a). The pure product was obtained by further crystallization from $95 \%$ ethanol. White needles of $\mathbf{1 a}(3.9 \mathrm{~g}, 90 \%)$ were obtained.

The second step of the synthesis was the Claisen cross-condensation between 1a $(5 \mathrm{mmol})$ and ethyl trifluoroacetate $(1.2 \mathrm{~mL})$ in the presence of a catalytic amount of potassium tert-butoxide. A solution of the reactants in dry toluene $(20 \mathrm{ml})$ was refluxed for $12 \mathrm{~h}$ under argon. After cooling, the mixture was treated with hydrochloric acid $(1 \mathrm{~mol} / \mathrm{L}, 30 \mathrm{~mL})$ and extracted with toluene. The solvent was evaporated under reduced pressure on a rotary evaporator. Next, the crude product was purified by column chromatography over silica gel and then recrystallized from ethanol to give pure 1-(9-ethylcarbazol-3-yl)-4,4,4-trifluorobutane-1,3-dione (1).
Spectroscopic analysis of 1-(9-ethylcarbazol-3-yl)ethanone (1a):

FTIR (KBr) [n, cm ${ }^{-1}$ : 3042, 2966, 1660, 1622, 1591, 1493, 1436, 1353, 1327, 1245, 1158, 748

${ }^{1} \mathbf{H}$ NMR $\left(300 \mathrm{MHz}, \mathrm{CDCl}_{3}\right) \delta 8.75(\mathrm{dd}, \mathrm{J}=1.7,0.5$ $\mathrm{Hz}, 1 \mathrm{H}), 8.21-8.08(\mathrm{~m}, 2 \mathrm{H}), 7.52(\mathrm{ddd}, \mathrm{J}=8.2,7.0$, $1.2 \mathrm{~Hz}, 1 \mathrm{H}), 7.48-7.37(\mathrm{~m}, 2 \mathrm{H}), 7.35-7.27(\mathrm{~m}, 1 \mathrm{H})$, $4.39(\mathrm{q}, \mathrm{J}=7.2 \mathrm{~Hz}, 2 \mathrm{H}), 2.88-2.57(\mathrm{~m}, 3 \mathrm{H}), 1.45(\mathrm{t}$, $\mathrm{J}=7.2 \mathrm{~Hz}, 3 \mathrm{H})$;

LC-ESI-MS: m/z $238.16[\mathrm{M}+\mathrm{H}]^{+}, 239.09[\mathrm{M}+2 \mathrm{H}]^{+}$.

Spectroscopic analysis of 1-(9-ethylcarbazol-3-yl)-4,4,4-trifluoro-butane-1,3-dione (1)

(mp $114-117^{\circ} \mathrm{C}$; lit.mp $114-115^{\circ} \mathrm{C}^{19}$ ):

FTIR $(\mathrm{KBr})\left[\mathrm{n}, \mathrm{cm}^{-1}\right]: 2974,1119,1613,1508,1457$, 1345, 1223, 1131, 1080, 1010, 929, 790, 745, 528;

${ }^{1} \mathbf{H}$ NMR $\left(300 \mathrm{MHz}, \mathrm{CDCl}_{3}\right) \delta 8.72(\mathrm{t}, J=7.7 \mathrm{~Hz}$, $1 \mathrm{H}), 8.22-8.12(\mathrm{~m}, 1 \mathrm{H}), 8.08(\mathrm{dd}, J=8.8,1.8 \mathrm{~Hz}$, $1 \mathrm{H}), 7.55(\mathrm{ddd}, J=8.2,7.1,1.2 \mathrm{~Hz}, 1 \mathrm{H}), 7.45(\mathrm{dd}, J=$ $8.4,6.3 \mathrm{~Hz}, 2 \mathrm{H}), 7.34(\mathrm{td}, J=7.5,1.0 \mathrm{~Hz}, 1 \mathrm{H}), 6.71(\mathrm{~s}$, $1 \mathrm{H}), 4.40(\mathrm{q}, J=7.2 \mathrm{~Hz}, 2 \mathrm{H}), 1.48(\mathrm{t}, J=7.2 \mathrm{~Hz}, 3 \mathrm{H})$;

LC-ESI-MS: $\mathrm{m} / \mathrm{z} 334.15[\mathrm{M}+\mathrm{H}]^{+}, 335.15[\mathrm{M}+2 \mathrm{H}]^{+}$, $352.09\left[\mathrm{M}+\mathrm{H}_{2} \mathrm{O}\right]^{+}$.

\section{Spectral measurements}

For the measurements of absorption and emission spectra, $6.6 \times 10^{-5} \mathrm{~mol} / \mathrm{dm}^{3}$ solutions of the compound $\mathbf{1}$ in various solvents were prepared. The absorption spectra of these solutions were recorded at room temperature, using the Genesys 20 Visible Spectrophotometer (Thermo Scientific) with a broadband tungsten-deuterium UV-Vis light source and a quartz cuvette with $1.0 \mathrm{~cm}$ optical path. For relative comparisons, the absorbance data were converted into extinction coefficient data, expressed in the classical units $\left[\mathrm{dm}^{3} \mathrm{~mol}^{-1} \mathrm{~cm}^{-1}\right]$. Fluorescence measurements were carried out using Shimadzu RF-5301 PC spectrofluorometer. All the fluorescence measurements were performed at right angle at room temperature using $1.0 \mathrm{~cm}$ thick quartz cuvette.

The absorption and emission spectra were measured in the following solvents: acetonitrile $(\mathrm{MeCN})$, acetone (AC), dimethylformamide (DMF), dimethylsulfoxide (DMSO), 1,4-dioksan (DO), diethyl ether (DEE), ethyl acetate $(\mathrm{EtOAc})$ and chloroform $(\mathrm{CHCl})$.

\section{Preparation of photocurable formulations}

The photocurable compositions were prepared by dissolution of the photoinitiator and each probe in the monomer, so as to obtain the concentrations $5.0 \cdot 10^{-3}$ $\mathrm{mol} / \mathrm{dm}^{3}$ of the probe and $1 \mathrm{wt} \%$ of the photoinitiator. The compositions were prepared in dimmed light, in vials made of brown glass, and were used shortly after the dissolution of the solid components in the monomer,

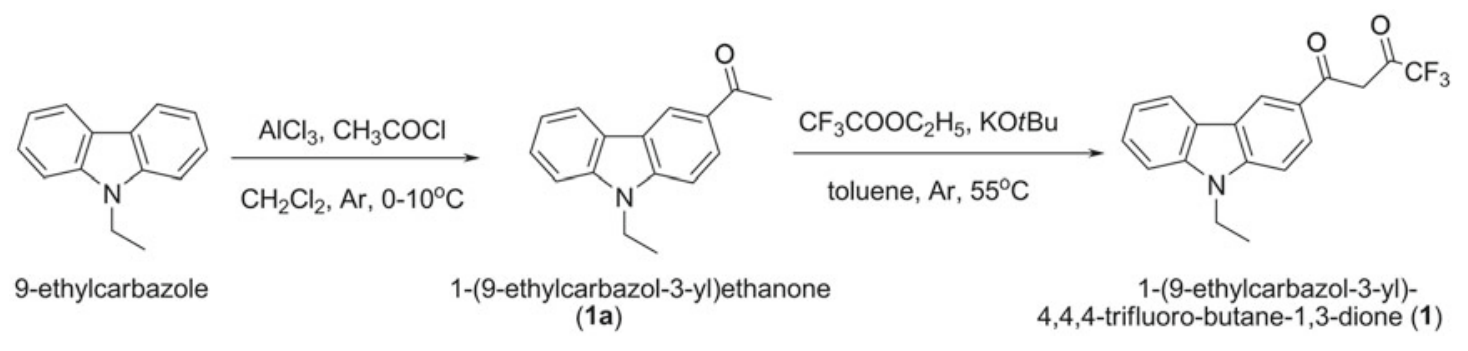

Figure 1. Synthesis of the probe 1 
because when the composition was exposed to daylight, spontaneous polymerization occurred.

\section{Preparation of thin-layer samples}

Before each measurement, a glass microscope slide (75 x $25 \times 1 \mathrm{~mm}$ ) was thoroughly cleaned and two rectangular spacers $(25 \times 15 \times 0.1 \mathrm{~mm})$ were placed on the slide sides. Next, two drops of the formulation tested were placed in the middle of the slide and another microscope slide was put on top. The formulation spread between the slides to form about $2-3 \mathrm{~cm}$ wide and $0.1 \mathrm{~mm}$ thick spot. The slides were kept at a constant distance with appropriate steel springs put on the sides where the spacers were located.

\section{FPT measurements}

The photopolymerization monitoring system was composed of a narrow-bandwidth UV light source, which was a UV LED emitting at the wavelength $\lambda_{\max }=320 \mathrm{~nm}$ (T9B31C, Seoul Optodevice, Korea), a miniature CCD spectrometer (EPP2000C, StellarNet Inc., USA) interfaced to a microcomputer, and an appropriate sensor head, where the sample was placed. For the transmission of fluorescence light from the measurement site to the spectrometer a fiber optic cable was applied. More details on the measurement system were previously described $^{5,6}$. All the measurements were done at ambient temperature $\left(20^{\circ} \mathrm{C}\right)$.

\section{RESULTS AND DISCUSSION}

Spectroscopic properties of 1-(9-ethylcarbazol-3-yl)-4,4,4-trifluorobutane-1,3-dione (1)

The absorption and fluorescence properties of probe 1 were investigated in 8 solvents of different physical and chemical properties at room temperature. The spectral data obtained are summarized in Table 1 , while the examples of excitation spectra are compared with the corresponding emission spectra in Figures 2 and 3. The normalized excitation and emission spectra of $\mathbf{1}$ in different solvents are also shown in Figures 4 and

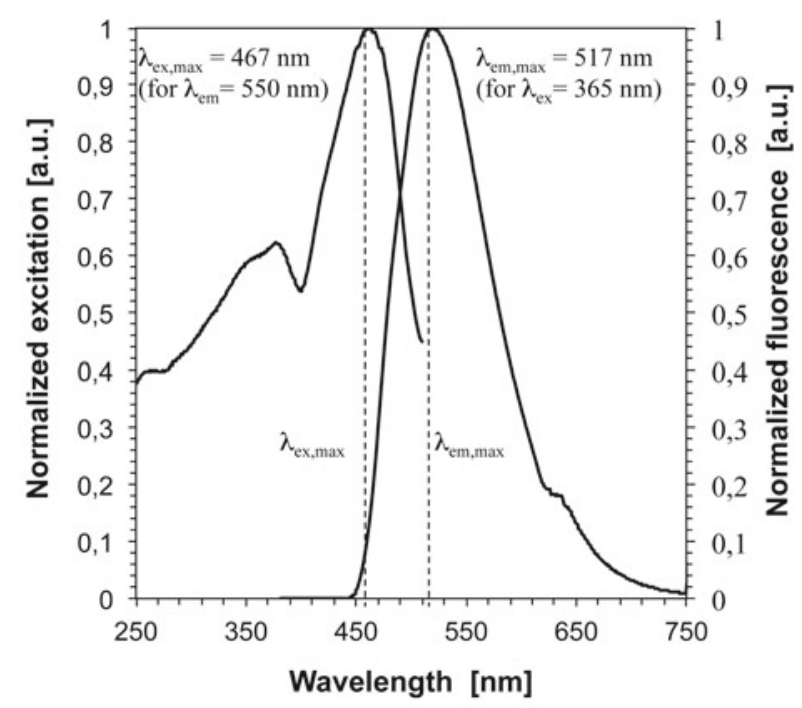

Figure 2. Excitation and fluorescence spectra of probe $\mathbf{1}$ in solid state

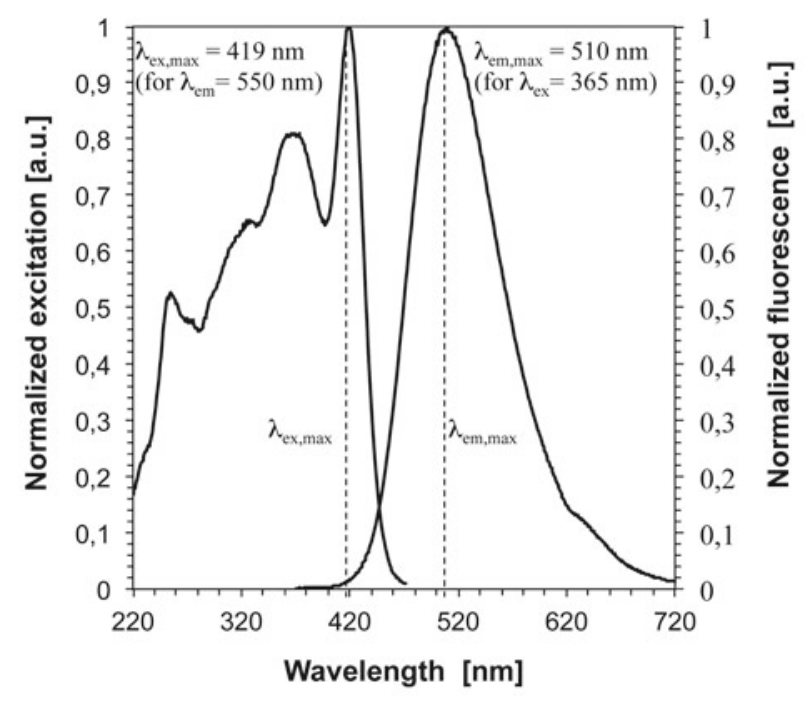

Figure 3. Excitation and fluorescence spectra of probe $\mathbf{1}$ in acetonitrile $(\mathrm{MeCN})$

Table 1. Steady state spectral properties of the fluorescent probe $\mathbf{1}$

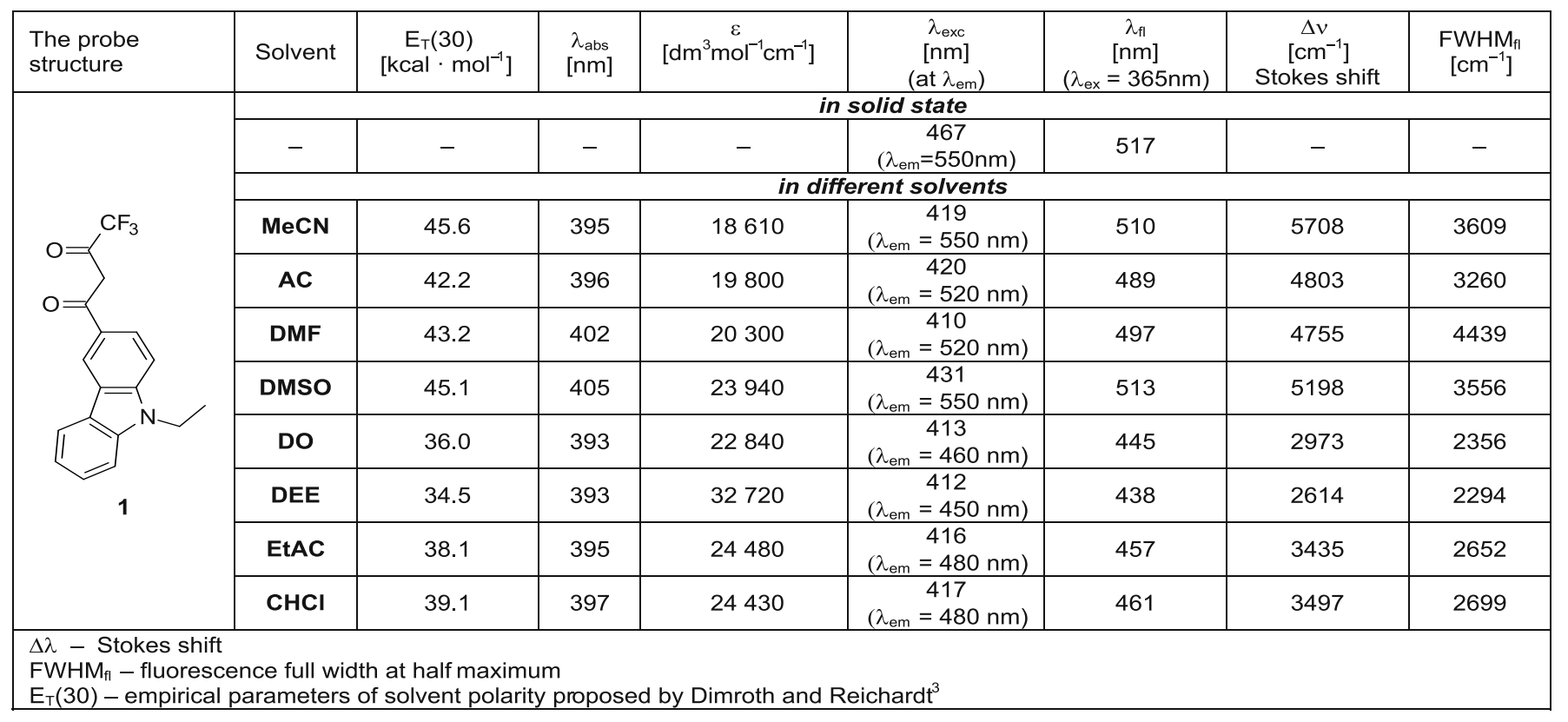


5 for the illustration of an interesting behavior of this compound in diverse environments. All the electronic excitation spectra of $\mathbf{1}$ display the main band maxima located in the range $400-470 \mathrm{~nm}$. In the case of the excitation spectra of $\mathbf{1}$, a relatively small shift $(\mathrm{Dl}=$ $19 \mathrm{~nm}$ ) is observed between the peak maxima in the least and the most polar solvent (Table 1 and Fig. 4.).

The fluorescence emission spectra of probe 1 recorded in various solvents appear in the range 400-680 $\mathrm{nm}$. As expected, the fluorescence maxima of $\mathbf{1}$ are significantly red-shifted in comparison with the absorption maxima (Table 1). The shift of emission spectra increases with the increase of the solvent polarity much more than the corresponding shift of the excitation maxima (Fig. 5 and Table 1) The longest emission wavelength was found in dimethylsulfoxide (DMSO, $1_{\max , \mathrm{em}}=513 \mathrm{~nm}$ ), while the shortest one was in diethyl ether $\left(\mathrm{DEE}, 1_{\max , \mathrm{em}}=438\right.$ $\mathrm{nm}$ ), which corresponds to the spectacular shift of 75 $\mathrm{nm}$ between the emission maxima, compared to only $19 \mathrm{~nm}$ in the case of excitation maxima. This indicates

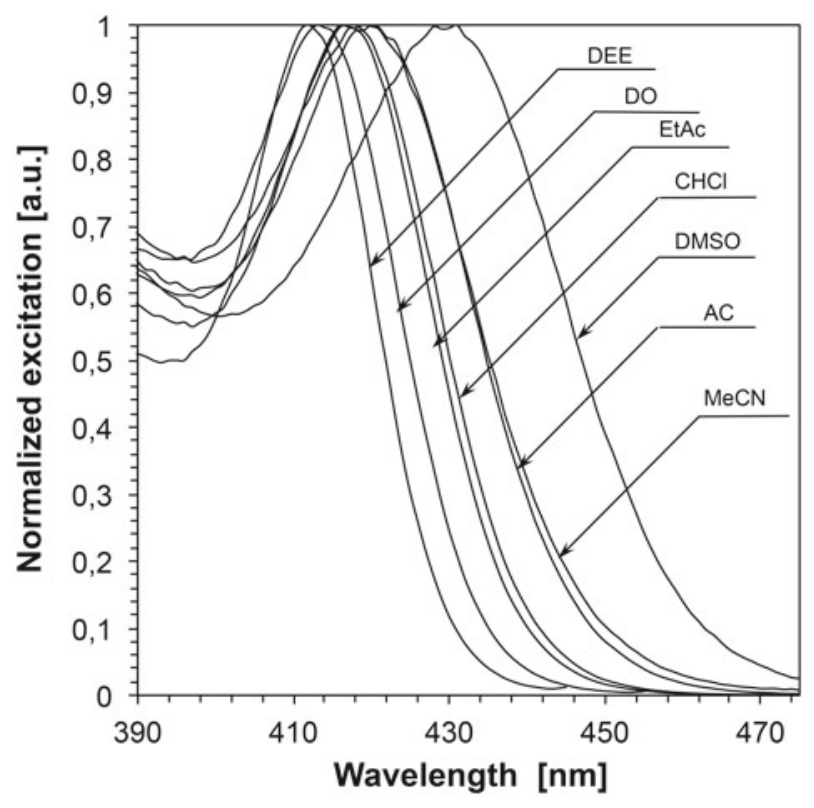

Figure 4. Excitation spectra of probe $\mathbf{1}$ in different solvents

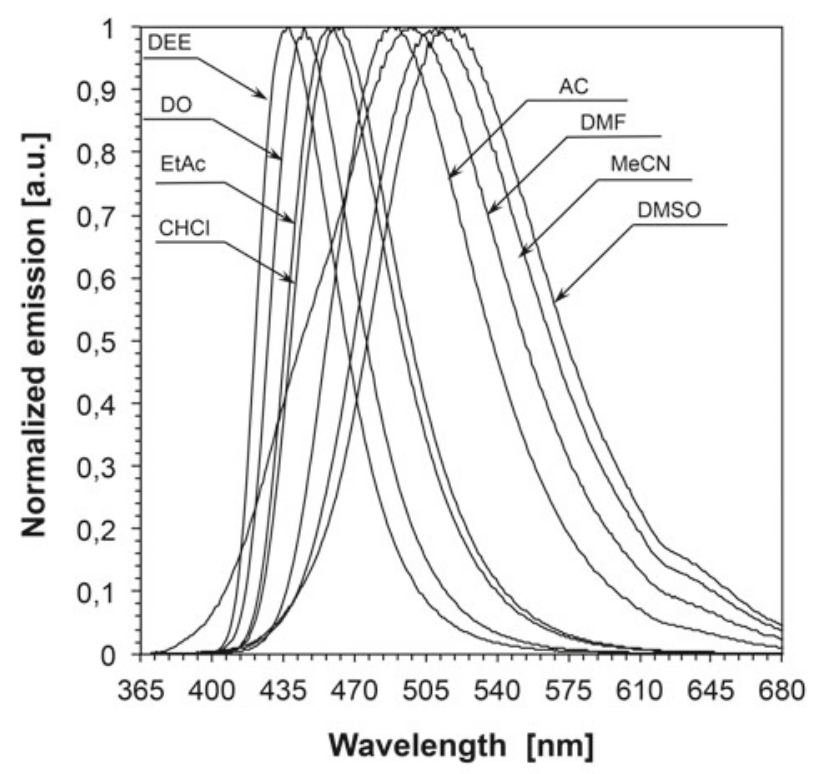

Figure 5. Emission spectra of probe $\mathbf{1}$ in different solvents that the solvent effect on the stabilization of the excited state of probe $\mathbf{1}$ is much greater than the effect on the probe ground state.

The correlation between the positions of the emission maxima of probe $\mathbf{1}$ and the Dimroth-Reichardt solvent polarity parameter $\mathrm{E}_{\mathrm{T}}(30)$ is shown in Figure 6 . Figure 6 indicates that the position of the emission maximum correlates very well with $\mathrm{E}_{\mathrm{T}}(30)$ parameter of the solvent. A large positive slope of the correlation suggests that an intramolecular charge transfer in the excited state of probe 1 plays a dominant role in the excited state behavior.

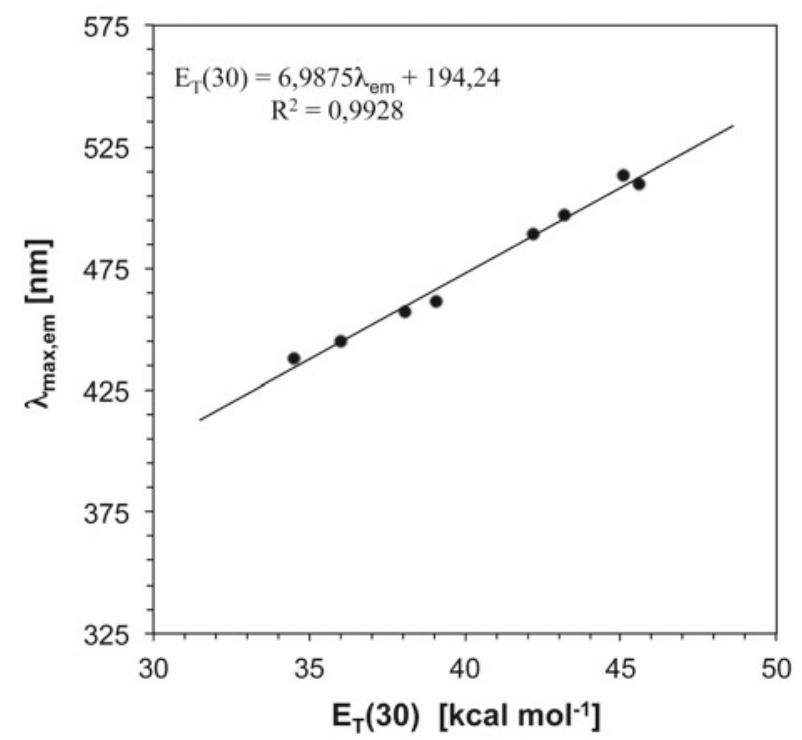

Figure 6. Correlation between the position of the emission maximum of robe $\mathbf{1}$ and the empirical solvent polarity parameter $\mathrm{E}_{\mathrm{T}}(30)$

Moreover, it is important to note that the high sensitivity of $\mathbf{1}$ to solvent polarity gives the possibility to use this carbazole derivative as a fluorescent molecular probe for monitoring photopolymerization processes.

\section{Monitoring of photopolymerization by FPT}

First, the fluorescence intensity ratio (R) was applied as an indicator of the polymerization progress. The $\mathrm{R}$ was defined as the ratio of fluorescence intensity at a shorter wavelength $\left(\lambda_{1}\right)$ to the intensity at a longer wavelength $\left(\lambda_{2}\right)$, both located on the opposite sides of the fluorescence spectrum maximum (Figures 7A and 7B). The monitoring wavelengths were determined individually for each probe from the probe fluorescence spectrum taken before monomer polymerization.

The wavelength $\lambda_{1}$ was selected in the middle of linear portion of the fluorescence spectrum on its short wavelength side, while $\lambda_{2}$ was selected so that the fluorescence intensity at $\lambda_{2}$ matched the intensity at $\lambda_{1}$. The so defined $\mathrm{R}$ values started from 1 and increased when the fluorescence spectrum shifted to shorter wavelengths. Figure 8 shows the progress of cationic photopolymerization of the composition studied, measured in real time, using the fluorescence intensity ratio (R) as the progress indicator. Probe 1 exhibits spectacular sensitivity to the changes occurring in its environment during the monomer polymerization. The sensitivity of $\mathbf{1}$ is about 2.3 times higher than that of probe 2 used as a 


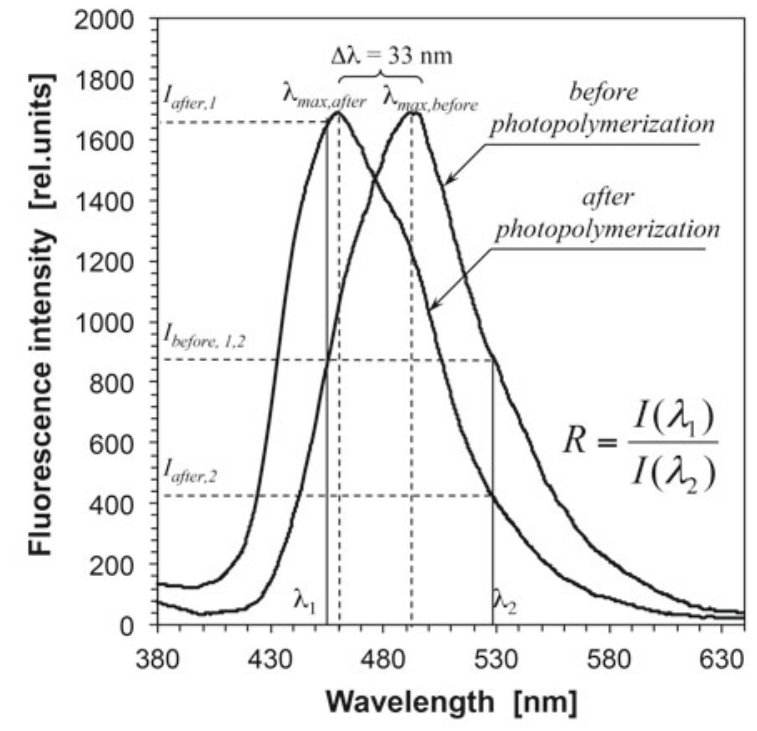

Figure 7a. Fluorescence spectra of probe 1 before and after cationic polymerization of TEGDVE monomer

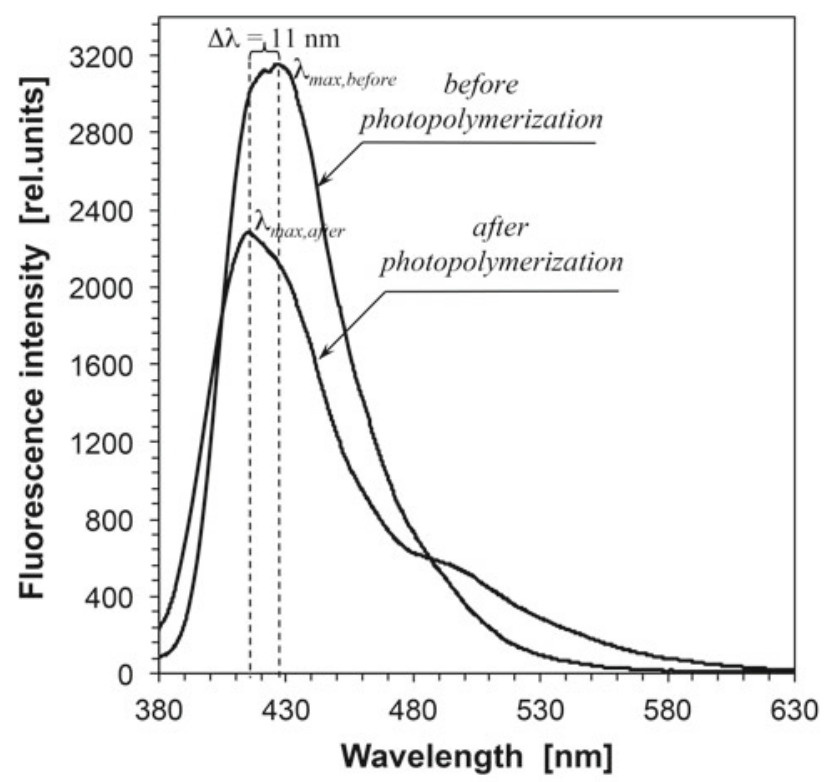

Figure 7b. Fluorescence spectra of Coumarin 1 before and after cationic polymerization of TEGDVE monomer

reference, as indicated by the magnitude of the ratio $(\mathrm{R})$ span between uncured and cured state. The increase of the ratio $(\mathrm{R})$ with the progress of monomer polymerization comes from the shift of the fluorescence spectrum towards shorter wavelengths (Fig. 8). Such shift during the monomer polymerization indicates that the system polarity decreased upon cure, which is typical for unsaturated monomers, because during the polymerization more polar double bonds of the monomer are converted to less polar single bonds in the polymer network. In the case of probe 1 this shift was $\mathrm{Dl}=33 \mathrm{~nm}$ in TEGDVE monomer (Fig. 7A), which can be considered as optimal for cure monitoring using the ratio $(\mathrm{R})$.

A larger shift would cause passing the fluorescence spectrum over its maximum at the wavelength $l_{1}$, which would cause a significant decrease of the probe sensitivity at high degrees of cure, because then both monitoring wavelengths would switch to the same side of the spectrum.

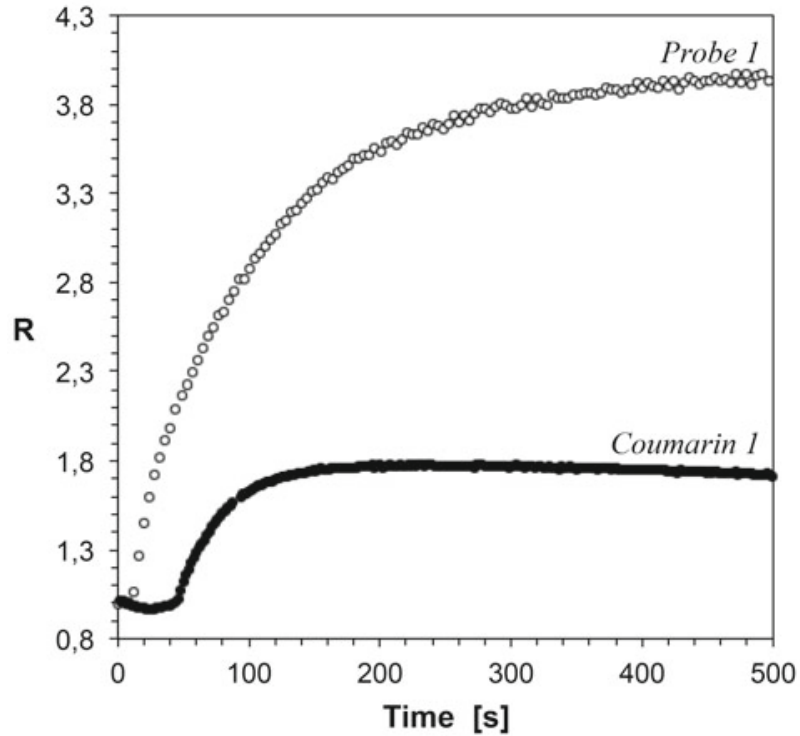

Figure 8. Kinetic profiles of cationic polymerization of TEGDVE monomer, obtained by the FPT method, using different probes and the fluorescence intensity ratio (R) as the polymerization progress indicator

Several other probes were previously developed for the purpose of the monitoring progress of cationic polymerization processes. Relative sensitivity of probe $\mathbf{1}$ in cationic polymerization of TEGDVE monomer is compared with the sensitivity of the other probes in the same monomer (though not necessarily using the same photoinitiator) in Table 2. It is evident from Table 2 that probe $\mathbf{1}$ is at least 2.5 -times more sensitive than any of the previous probes.

Finally, it can be observed that probe 2 exhibits a distinct induction period of the order of $40 \mathrm{~s}$ during

Table 2. Comparison of the relative sensitivity of different probes suitable for the monitoring progress of cationic photopolymerization of TEGDVE monomer

\begin{tabular}{|l|l|l|}
\hline Relative sensitivity, & Reference \\
{$[\%]^{*}$}
\end{tabular}

${ }^{*}$ Relative sensitivity $=\left(R_{\max }-R_{0}\right) / R_{0} \cdot 100 \%$, where $R_{\max }$ - the ratio after polymerization, $R_{o}$ - the inital ratio before polymerization. 
cationic photopolymerization of TEGDVE, while the corresponding delay of the polymerization start was five times shorter in the case of probe $\mathbf{1}$ (Fig. 8). This is consistent with much weaker basicity of probe 1 compared to that of probe 2 . In each case, after the induction period, where any contaminants present in the system were protonated (including the diethylamino group of probe 2) and sufficient amount of hexafluorophosphoric acid was generated from the photoinitiator, cationic polymerization began, as indicated by a sharp increase of the ratio $(\mathrm{R})$. When the polymerization process ended, the response of probes $\mathbf{1}$ and $\mathbf{2}$ leveled off, as expected.

\section{CONCLUSION}

1-(9-ethylcarbazol-3-yl)-4,4,4-trifluorobutane-1,3-dione (1) shifts its fluorescence spectrum to a shorter wavelength upon cationic polymerization of the medium, which enables monitoring the polymerization progress using the fluorescence intensity ratio $(\mathrm{R})$ as the progress indicator. This probe is stable enough under the cationic polymerization conditions of vinyl ethers and affords correct response even at high monomer conversions. The response of the carbazole probe $\mathbf{1}$ is much more sensitive to changes occurring in its environment than that of the coumarin probe $\mathbf{2}$ and that of the other fluorescent probes developed for the purpose of monitoring cationic polymerization. For this reasons probe $\mathbf{1}$ is applicable for use as a new highly sensitive fluorescent probe for cure monitoring of cationic polymerization processes by the FPT method both off-line and on-line within a broad range of monomer conversions.

\section{ACKNOWLEDGEMENT}

This research was supported by the Ministry of Science and Higher Education project: Iuventus Plus - 0394/ IP3/2011/71. This support is gratefully acknowledged.

The work was also supported by the European Union through the European Social Fund within \&\#8222;Cracow University of Technology development program - top quality teaching for the prospective Polish engineers; University of the 21st century\&\#8221; project (contract no.UDA-POKL.04.01.01-00-029/10-00).

\section{LITERATURE CITED}

1. Zhang, J., Campbell, R.E., Ting, A.Y. \& Tsien, R.Y. (2002). Creating new fluorescent probes for cell biology. Nat. Rev. Mol. Cell. Biol. 3(12), 906-918. DOI: 10.1038/nrm976.

2. Goncalves, M.S.T. (2009). Fluorescent labeling of biomolecules with organic probes. Chem. Rev.109(1), 190-212. DOI: $10.1021 / \mathrm{cr} 0783840$.

3. Reichardt, Ch. (1994). Solvatochromic dyes as solvent polarity indicators. Chem. Rev. 94, 2319-2358. DOI: 10.1021/ cr00032a005.

4. Haidekker, M.A., Brady, T.P., Lichlyter, D. \& Theodorakis, E.A. (2005). Efects of solvent polarity and solvent viscosity on the fluorescent properties of molecular rotors and related probes. Bioorg.Chem. 33(6), 415-425. DOI: 10.1016/j. bioorg.2005.07.005.

5. Ortyl, J., Sawicz, K. \& Popielarz, R. (2010). Performance of amidocoumarins as probes for monitoring of cationic photopolymerization of monomers by fluorescence probe technology. J. Polym. Sci., Part A: Polym. Chem. 48(20), 4522-4626. DOI: 10.1002/pola.24243.
6. Ortyl, J., Galek, M., Milart, P. \& Popielarz, R. (2012). Aminophthalimide probes for monitoring of cationic photopolymerization by Fluorescence Probe Technology and their effect on the polymerization kinetics. Polymer Testing. 31(3), 466-473. DOI:10.1016/j.polymertesting.2012.01.008.

7. Hu, S., Popielarz, R. \& Neckers, D.C. (1998). Fluorescence Probe Techniques (FPT) for measuring the relative efficiencies of free-radical photoinitiators. Macromolecules 31, 4107-4113. DOI: $10.1021 / \mathrm{ma} 9713901$.

8. Vatanparast, R., Li, S., Hakala, K. \& Lemmetyinen, H. (2000). Monitoring of curing of polyurethane polymers with fluorescence method. Macromolecules, 33, 438-443. DOI: 10.1021/ma991414j.

9. Itagaki, H., Horie, K. \& Mitra, I. (1990). Luminescent probe studies of the microstructure and mobility of solid polymers. Prog. Polym. Sci., 15(3), 361-424. DOI: 10.1016/00796700(90)90002-I.

10. Ortyl, J., Sawicz-Kryniger, K. \& Popielarz, R. (2011). Mechanism of Coumarin 1 probe response in fluorescence probe technology (FPT). Przem. Chem. 90(7), 1370-1378.

11. Bosch, P., Catalina, F., Corrales, T. \& Peinado, C. (2005). Fluorescent probes for sensing processes in polymers. Chem. Eur. J.. 11, 4314-4325. DOI: 10.1002/chem.200401349.

12. Ortyl, J., Galek, M., Sawicz-Kryniger, K. \& Popielarz, R. (2010). Monitoring of cationic photopolymerization with stilbene derivatives as fluorescent probes. Przem. Chem. 89(12): 1642-1646.

13. Xiang-Dong Sun \& Chong Sook Paik Sung. (1996). Cure characterization in polyurethane and model urethane reactions by an intrinsic fuorescence technique. Macromolecules. 29, 3198-3202. DOI: 10.1021/ma9515339.

14. Schaeken, T.C. \& Warman, J.M. (1995). Radiation-induced polymerization of a mono- and diacrylate studied using a fluorescent molecular probe. J. Phys. Chem. 99, 6145-6151. DOI: $10.1021 / \mathrm{j} 100016 \mathrm{a} 059$.

15. Ortyl, J. \& Popielarz, R. (2013). The performance of 7-hydroxycoumarin-3-carbonitrile and 7-hydroxycoumarin3-carboxylic acid as fluorescent probes for monitoring of cationic photopolymerization processes by FPT, J. Appl. Polym. Sci. 128 (3), 1974-1978. DOI: 10.1002/app.38378.

16. Wang, J.Z., Song, J.C., Rong Bao \& Neckers, D.C. (1996). Fluorescence probes for monitoring polymerization processes. J. Polym. Sci. Part B. 34(2), 325-333. DOI: 10.1002/(SICI)10990488(19960130)34:2<325::AID-POLB13>3.0.CO;2-L.

17. Sawicz, K., Ortyl, J. \& Popielarz, R. (2010). Applicability of 7-hydroxy-4-methylcoumarin for cure monitoring and marking of epoxy resins. Polimery 55(7-8), 539-544.

18. Okay, O., Kaya, D. \& Pekcan, O. (1999). Free-radical crosslinking polymerization of styrene and divinylbenzene: real time monitoring of the gel effect fluorescence probe. Polymer. 40, 6179-6187. DOI:10.1016/S0032-3861(98)00831-3.

19. Bowyer, P.M., Iles, D.H. \& Ledwith, A. (1971). Chlorination of carbazole and its derivatives with 1-chlorobenzotriazole. J. Chem. Soc. C, 2775-2777. DOI: 10.1039/J39710002775.

20. He, P., Wang, H., Liu, S., Shi, J., Wang, G. \& Gong, M. (2009). Effect of different alkyl groups at the $N$-position on the luminescence of carbazole-based $\beta$-diketonate europium(III) complexes. J. Phys. Chem. A. 113(46), 12885-12890. DOI: 10.1021/jp908416q.

21. Ortyl, J., Sawicz, K., Popielarz, R., Galek, M., Milart, P. (2010). Applicability of selected biphenyl derivatives for monitoring of cationic photopolymerization by Fluorescent Probe Technology. Luminescence, 25, 225-226.

22. Ortyl, J., Popielarz, R. (2012). New photoinitiators for cationic polymerization. Polimery, 57, 510-517. 